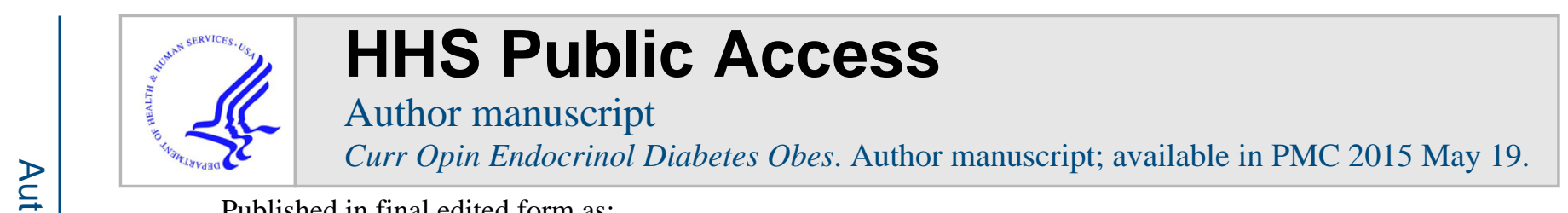

Published in final edited form as:

Curr Opin Endocrinol Diabetes Obes. 2015 February ; 22(1): 41-47. doi:10.1097/MED.

0000000000000125.

\title{
Sleep patterns and obesity in childhood
}

\author{
Alison L. Miller ${ }^{a, b}$, Julie C. Lumeng ${ }^{a, b, c}$, and Monique K. LeBourgeois ${ }^{d}$ \\ aSchool of Public Health, University of Michigan, Ann Arbor, Michigan

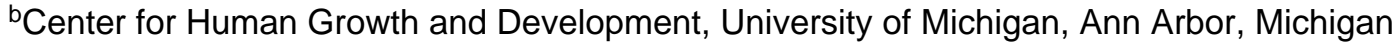 \\ 'Department of Pediatrics, University of Michigan, Ann Arbor, Michigan \\ dDepartment of Integrative Physiology, University of Colorado, Boulder, Colorado, USA
}

\begin{abstract}
Purpose of review-To highlight the recent findings on sleep-obesity associations in children. We focus on sleep duration, sleep timing and chronotype, and describe the potential mechanisms underlying sleep-obesity associations.
\end{abstract}

Recent findings-Poor sleep is increasingly common in children and associations between short sleep duration in early childhood and obesity are consistently found. Less is known about the infancy period, and the findings in adolescents are inconsistent. Sleep timing patterns may also contribute to obesity risk. Variable and shifted sleep schedules and evening chronotypes have recently been linked to adiposity in adults; less is known about children. Further, there is little understanding regarding the mechanisms of association. The timing of eating, dietary intake, obesogenic eating behaviors, and changes in appetite-regulating hormones have been identified as possible mechanisms for sleep-obesity associations and may be promising avenues for future research. Longitudinal and experimental work with children is needed to determine the nature of associations.

Summary-Beyond sleep duration, sleep timing patterns may contribute to obesity risk. Biological and behavioral processes have been proposed as mechanisms that may explain the association. Understanding the pathways through which poor sleep patterns could increase obesity risk in children may provide novel avenues for intervention.

\section{Keywords}

BMI; child obesity; chronotype; eating behavior; sleep timing

Copyright @ 2015 Wolters Kluwer Health, Inc. All rights reserved.

Correspondence to Alison L. Miller, PhD, Department of Health Behavior and Health Education, University of Michigan School of Public Health, 1415Washington Heights, SPH I, University of Michigan, Ann Arbor, MI 48109-2029, USA. Tel: +1 734 615 7459; fax: +1 734615 2379; alimill@umich.edu.

Conflicts of interest

None. 


\section{INTRODUCTION}

Obesity is widespread in the USA, with a prevalence rate of $35 \%$ in adults and $17 \%$ in children [1]. Short sleep duration is also common [2] - more than $30 \%$ of adults sleep less than $6 \mathrm{~h}$ per night [3], only $20 \%$ of adolescents get their optimal $9 \mathrm{~h}$ of sleep on school nights, and one-third of 2-3 year olds sleep less than recommended [4]. An association between short sleep duration and obesity or adiposity during early childhood has emerged in numerous epidemiological studies over the last decade; however, sleep-obesity associations at other points in development are less well studied. Further, understanding the multiple aspects of sleep patterns, rather than sleep duration alone, may identify the contributing mechanisms and provide new opportunities for sleep-focused interventions to prevent obesity. This review will provide updates in three areas, focusing on studies published since 2013: sleep duration-obesity associations during infancy and adolescence; associations of sleep timing and chronotype with obesity; and selected biobehavioral mechanisms of association between poor sleep patterns and obesity risk.

\section{DEVELOPMENTAL DIFFERENCES IN SLEEP DURATION-OBESITY ASSOCIATIONS}

Studies have consistently identified an association between short sleep during early childhood (age 3-7 years) and either concurrent or later obesity (see [5] for review). In comparison, a gap exists in understanding the sleep duration in relation to adiposity during infancy and toddlerhood (prior to age 3 years), even though sleep problems and sleep loss are common at this age [4]. A recent longitudinal study found that chronic short sleep from infancy through school age was associated with increased adiposity indicators and obesity [6-r]; associations between short sleep in infancy and adiposity at age 3 years had been previously reported in this cohort [7]. Similar to most epidemiological studies, this analysis relied on parent-reported sleep. Results from the few studies using actigraphy to measure infant sleep have been mixed: one study found the expected association between short nighttime (though not daytime) sleep and greater weight-for-length at age 6 months [8], and another found no association between infant sleep (parent reported at 9 and 18 months, actigraphy at 36 months) and adiposity at 36 months [9m]. Incorporating the objective measurements of sleep such as actigraphy in future work may be important in clarifying the nature of the association (if any) and better articulate the mechanism underlying earlyemerging sleep-obesity associations.

Later school age and adolescent periods also remain understudied compared with early childhood, despite adolescents' known short sleep duration because of interactions between sleep bio-regulatory processes (i.e., circadian phase delay and attenuation of the buildup of sleep pressure) and external constraints such as school start times [10]. Studies of sleep duration-obesity associations during adolescence have yielded mixed results, perhaps in part because of the methodological differences (see [11-] for a recent review). A recent crosssectional study found short sleep-adiposity associations for both boys and girls at age 9 [12--] and 10 years [13-], whereas a longitudinal study of 9-11 year olds found associations between short sleep and BMI for girls, but not for boys [14--]. Among older adolescents, a cross-sectional study has shown the expected inverse relationship between short sleep 
duration and adiposity, with frequent sex differences. For example, the association was present for younger boys (age 13 [15] and 16 years $[16-17 \cdot]$ ) and to some degree for older girls (age 17 years [15]). Longitudinal studies across this age range have been inconclusive. In boys, short sleep at 13 years predicted higher BMI $z$-score at age 17 [15], though no association was observed for boys or girls between changes in sleep duration and changes in adiposity across age 14-18 years in another study [18-]. Still another study found that short sleep at age 14 predicted weight gain across age 14-18 years, with the strongest associations found among heavier adolescents [19--]. Thus, the mechanisms through which short sleep may confer obesity risk during adolescence are complex and may differ from the early childhood period. Longitudinal work that can identify sleep-growth associations at multiple timepoints is vital (see [20--]). Furthermore, as child sex may moderate these associations during the adolescent period, it will be important to consider and assess pubertal status in future work.

\section{BEYOND SLEEP DURATION: SLEEP TIMING AND PATTERN}

Sleep timing is emerging as an important mechanism through which poor sleep patterns may confer obesity risk in adults [21], but less is known about sleep timing-obesity associations in infants, children, or adolescents. Aspects of poor sleep timing include late bedtimes and more variable patterns, including variability throughout the week and between weekday and weekend sleep schedules, which indicate a 'shifted' or 'delayed' sleep phase.

\section{Late bedtimes}

Two reports in the last year specifically examined sleep timing and obesity in early childhood, finding that late bedtimes (after 9 p.m.) magnified [22--] and independently predicted [23--] the association between short sleep duration and obesity. Studies of schoolage children and adolescents (age 8-17 years) also found that late bedtimes were associated with adiposity, independent of sleep duration [24--25--]. In a small study of obese 13 year olds $(n=26)$, late bedtimes were related to increased cumulative energy intake and more screen time, even though total sleep duration was the same for 'late-sleeping' (mean bedtime 10 p.m., mean waketime 7 a.m.) and 'early-sleeping' (mean bedtime 11 p.m., mean waketime 8 a.m.) groups [26--]. Although this finding needs to be replicated in a larger sample, it suggests that late bedtime may be a unique contributor to obesity risk.

\section{Variable and shifted schedules}

Earlier work found that greater weekday-to-weekend variability in sleep timing, in combination with shorter sleep duration, was associated with obesity and poorer metabolic health in 4-10-year-old children [27], and that school-age children and adolescents (9-16 years) demonstrating a late-bedtime/late-rise time pattern were more likely than those with an early-bedtime/early-rise time pattern to be overweight and engage in more screen time and less physical activity [28]. More recently, variability between weekend and weekday sleep schedules has been associated with adiposity in school-age children and adolescents, independent of sleep duration. One study [29] found that more overweight adolescents (14 years of age) had late weekend compared with weekday bedtimes, but there was no concurrent association between sleep duration and weight status. In slightly younger 
children (12 years of age), oversleeping on the weekends was associated with increased adiposity, and the association with sleep duration was attenuated after accounting for sleep timing [25-r]. A study of 8-11-year-old Danish children found that more variable sleep timing during a given week was associated with greater energy intake, independent of total sleep duration, screen time, and demographic confounders [30--].

\section{Sleep timing and obesity risk: complex associations across development}

If sleep timing patterns contribute to obesity risk independent of sleep duration, identifying when such associations first emerge is important in determining when and how to intervene. Very young children meet their 24-h sleep need in part via daytime naps [2], for example, yet most studies examining daytime napping in relation to adiposity in young children have not found the associations (e.g., [22--]). As children transition to formal school settings (e.g., kindergarten), their sleep patterns change; children as young as age 5 years may 'shift' their bedtimes late on weekends compared with weekdays [31]. Maintaining a regular and early bedtime schedule during the week, as well as across weekdays and weekends, may be an important obesity prevention strategy by reducing the behavioral and metabolic changes that occur as a result of shifted sleep timing [32,33-]. This strategy may be particularly critical for school-age children and adolescents, who can face challenges to optimal sleep timing; yet, considering how such sleep timing patterns may relate to obesity risk in younger children is also important.

\section{CHRONOTYPE: AN UNDERLYING DIURNAL PREFERENCE THAT MAY SHAPE TIMING OF SLEEPING, EATING, AND ACTIVITY PATTERNS RELEVANT FOR OBESITY}

It has recently been suggested that 'chronotype', a construct reflecting individual differences in diurnal preference (e.g., being an early bird or a night owl), may independently contribute to obesity risk by influencing the time(s) of day when an individual is more likely to sleep, eat, or be physically active [34--36-- ]. In addition, 'social jetlag' (i.e., weekday sleep restriction followed by a weekend delayed sleep phase) has been proposed as a direct cause of obesity through metabolic changes [32] and indirectly through behavioral pathways such as eating behavior $[35-\mathbf{m}, 36-\mathbf{m}, 37]$. A social jetlag pattern may arise from repeatedly shifting sleep timing, and is characterized by a misalignment of circadian-regulated sleep and appetite that can disrupt the underlying metabolism [32,33-]. Studies of college students and adults have found that a preference for evening alertness and late sleeping was associated with unhealthy diet [35--,38] and weight gain [35--,39-]. Furthermore, among adolescents (11-16 years) evening chronotype was also associated with higher BMI [34--,36--] and more unhealthy eating behaviors [34- 37].No study has examined the emergence of these associations in younger children; however, recent work [40--] determined that parent reports of chronotype were associated with a reliable marker of the timing of the circadian clock, salivary dim light melatonin onset. The early emergence of chronotype and associated sleep, eating, and activity timing patterns as they relate to childhood obesity risk is an exciting new research direction. 


\section{BIOLOGICAL AND BEHAVIORAL MECHANISMS PROPOSED TO UNDERLIE THE SLEEP-OBESITY ASSOCIATION}

The mechanisms linking sleep and obesity are not fully understood, and a comprehensive update on all such relevant findings is beyond the scope of this review. Recent experimental work on the biological and behavioral mechanisms underlying sleep-obesity associations, including some studies with children, has nonetheless uncovered intriguing possibilities that could greatly inform new research and intervention efforts. We focus on the studies that consider how the timing of sleep and eating behaviors, in addition to sleep duration (or restriction) alone, may contribute to these pathways.

\section{Biological mechanisms}

For over a decade, short sleep duration has been suggested to lead to increased weight gain through hormonal changes associated with appetite regulation, specifically leptin and ghrelin secretion (e.g., [41]). Much less is known about how such processes operate in children, and recent work has found conflicting results. One large epidemiological study showed that chronically short sleep duration in school-age children was associated with lower leptin levels [42-m], whereas a smaller study that experimentally manipulated sleep duration found higher leptin levels associated with short sleep [43--]. Methodological differences across the studies may account for different results, and neither study considered sleep timing or circadian cycle, which can regulate metabolism [44-]. Thus, it may be that sleep timing and chronotype as well as sleep duration shape the physiological processes relevant for weight gain [45-]. Investigating how sleep loss and sleep timing patterns may interact and independently contribute to the biological changes that could increase obesity risk, particularly in children, is an area ripe for research.

\section{Behavioral mechanisms}

Although appetite-regulating hormones and other biologically mediated pathways have been the focus of significant research, it has been recently noted that adults tend to increase their energy intake after sleep restriction even in the absence of hormonal changes [46-,47--[], suggesting the importance of behaviorally mediated pathways. Although multiple behavioral mechanisms such as screen time and physical activity are clearly important, we focus here on the pathways related to eating behavior.

\section{Dietary intake and quality}

Experimental studies in adults have shown that increased energy consumption is a primary mechanism for weight gain following sleep restriction [48--,49-50]. Experimental work with very young children has not been conducted. Yet, shorter nocturnal sleep at 16 months of age was associated with greater intake of energy 5 months later in a large birth cohort study [51-m]. Observational work with adolescents has also found associations between short sleep duration and poor dietary quality [52-]. The few extant experimental studies with children are consistent with such results, finding that adolescents ate more dessert after five nights of sleep restriction [53--], and that school-age children consumed more calories overall the day following sleep restriction [43--]. Understanding how dietary intake may be 
altered as a result of sleep restriction (or extension) in young children should be further investigated in future work using experimental designs [46-].

\section{Timing of eating}

Behavioral mechanisms that promote obesity may be related to what time during the day children sleep, eat, and engage in sedentary versus active activities. Sleep timing and chronotype may thus contribute to obesity in part through shaping the timing of eating behavior $[45 \cdot, 54 \cdot, 55 m, 56]$. Snacking outside of mealtimes has increased in recent years and is proposed as a contributor to excessive weight gain in children [57-]. Young children may also wake at night and eat to soothe themselves back to sleep, and seek to delay bedtime by asking for food. Yet, little is known about how the timing of sleep and the timing of eating behavior relate to obesity risk in children; in one observational study, overweight school-age chldren ate more later in the day, whereas overweight adolescents ate less [58]. In experimental work with adults, participants who were sleep-restricted compared with well rested gained weight and consumed more calories specifically because of eating late at night [48- $-49-50]$, with foods eaten during the evening more likely to be high in carbohydrates [48-,50] and fat, salt, and sugar [59-]. Correlational studies have also found an association between adiposity indicators and the late-night eating of fats and carbohydrates [60-].

\section{Obesogenic eating behavior}

Finally, dietary intake is the outcome of several increasingly well characterized obesitypromoting eating behaviors such as lack of satiety responsiveness, food responsiveness, enjoyment of food, emotional eating, eating in response to external versus internal cues, and restrained eating [61,62-m]. Recent work suggests that such eating behaviors may moderate or mediate the association between short sleep duration and obesity. In a small study of children (age 5-12 years), short sleep duration, poor sleep quality, and late bedtimes were associated with emotional eating, eating in response to external cues, and restrained eating respectively [63-], suggesting that such eating behaviors may mediate sleep-obesity associations. Among adult women, eating behavior moderated the association, such that the effect of short sleep on food intake was greater for women who reported being emotional eaters [64]. Articulating the role of such eating behaviors is an important direction for future work.

\section{CONCLUSION}

Identifying how sleep patterns (i.e., timing and chronotype), above and beyond the sleep duration, may contribute to obesity risk across different developmental periods can help us understand the longitudinal and mechanistic associations and design effective interventions. Establishing a regular, consistent sleep schedule early in childhood may enhance metabolic regulation and prime healthier sleep and eating habits in the future. Sleep schedules shift increasingly with development and implications of sleep timing for obesity appear to begin at least during early childhood [20--], a time when children shift from a biphasic to amonophasic sleep schedule [31]. Adolescents may be particularly vulnerable to the effects of poor sleep timing, perhaps as a function of their sex and pubertal status. Experimental work with adults has articulated specific biological and behavioral pathways connecting 
sleep and obesity, many of which appear to operate through the timing of sleep and eating behavior, and mechanistic research on how such processes operate in children is critical.

\title{
Acknowledgements
}

\author{
None. \\ Financial support and sponsorship
}

A.L.M and J.C.L. are supported by the grants NICHD/NIDDK R01DK095695, NICHD R01HD061356, and NICHD 1R01HD069179.

A.L.M. and M.K.L. are supported by the grant NIMH R01MH086566.

\section{REFERENCES AND RECOMMENDED READING}

Papers of particular interest, published within the annual period of review, have been highlighted as:

- of special interest

- of outstanding interest

1. Ogden CL, Carroll MD, Kit BK, Flegal KM. Prevalence of childhood and adult obesity in the United States. JAMA. 2014; 311:806-814. [PubMed: 24570244]

2. Iglowstein I, Latal Hajnal B, Molinari L, et al. Sleep behaviour in preterm children from birth to age 10 years: a longitudinal study. Acta Paediatr. 2006; 95:1691-16933. [PubMed: 17129987]

3. National Center for Health Statistics. QuickStats: percentage of adults who reported an average of $<6 \mathrm{~h}$ of sleep per 24-h period, by sex and age group - United States, 1985 and 2004. MMWR. 2005; 54:933.

4. National Sleep Foundation. Sleep in America Poll. Washington, D.C: National Sleep Foundation; 2006.

5. Patel SR, Hu FB. Short sleep duration and weight gain: a systematic review. Obesity. 2008; 16:643653. [PubMed: 18239586]

6. Taveras EM, Gillman MW, Pena MM, et al. Chronic sleep curtailment and adiposity. Pediatrics. 2014; 19:2013-3065. A longitudinal cohort study examining sleep and adiposity in low-income children from infancy to school age, the only study to examine infant sleep in relation to adiposity in large cohort.

7. Taveras EM, Rifas-Shiman SL, Oken E, et al. Short sleep duration in infancy and risk of childhood overweight. Arch Pediatr Adolesc Med. 2008; 162:305-311. [PubMed: 18391138]

8. Tikotzky L, De Marcas G, Har-Toov J, et al. Sleep and physical growth in infants during the first 6 months. J Sleep Res. 2010; 19(1-Part-I):103-110. [PubMed: 19840242]

9. Klingenberg L, Christensen LB, Hjorth MF, et al. No relation between sleep duration and adiposity indicators in 9-36 months old children: the SKOT cohort. Pediatr Obes. 2013; 8:2047-6310. The only study to date to objectively assess infant sleep duration (at 9,18 , and 36 months) and adiposity (36 months); this study found no association.

10. Carskadon MA. Sleep in adolescents: the perfect storm. Pediatr Clin North Am. 2011; 58:637-647. [PubMed: 21600346]

11. Thind H, Davies SL, Lewis T, et al. Does short sleep lead to obesity among children and adolescents? Current understanding and implications. Am J Lifestyle Med. 2014 [Epub ahead of print]. A review of the longitudinal studies of sleep duration and obesity in adolescents; this study discusses the sex differences and methodological measurement issues that lead to inconsistent findings.

12. Martinez SM, Greenspan LC, Butte NF, et al. Mother-reported sleep, accelerometer-estimated sleep and weight status in over 300 Mexican American children: sleep duration is associated with 
increased adiposity and risk for overweight/obese status. J Sleep Res. 2014; 23:328-336. A crosssectional study of 10-year-old Mexican-American children using actigraphy and maternal-reported measures of sleep; objective sleep assessment was more strongly related to BMI, but maternal reports were acceptable.

13. Pileggi C, Lotito F, Bianco A, et al. Relationship between chronic short sleep duration and childhood body mass index: a school-based cross-sectional study. PLoS One. 2013; 8:e66680. [PubMed: 23805261] A cross-sectional study of over 500 10-year-old Italian children; this study found association between parent-reported short sleep and BMI.

14. El-Sheikh M, Bagley EJ, Keiley MK, Erath SA. Growth in body mass index from childhood into adolescence: the role of sleep duration and quality. J Early Adolesc. 2014; 134:1145-1166. A longitudinal study of 9-11-year-old U.S. children; this study objectively measured sleep duration was associated with BMI for girls, but not boys $(n=273)$.

15. Araújo J, Severo M, Ramos E. Sleep duration and adiposity during adolescence. Pediatrics. 2012; 130:e1146-e1154. [PubMed: 23027175]

16. Suglia SF, Kara S, Robinson WR. Sleep duration and obesity among adolescents transitioning to adulthood: do results differ by sex? J Pediatr. 2014; 165:750-754. [PubMed: 25066064] A longitudinal study of sleep duration and BMI from adolescence across the transition to adulthood using New York Longitudinal Study of Youth $(n>10000)$; mixed associations during adolescence but short sleep duration during adolescence predicted higher BMI in young adulthood.

17. Skidmore PM, Howe AS, Polak MA, et al. Sleep duration and adiposity in older adolescents from Otago, New Zealand: relationships differ between boys and girls and are independent of food choice. Nutr J. 2013; 12:128-138. [PubMed: 24034352] A cross-sectional study of sleep duration and body composition in adolescents; associations were found only for boys and only for fat mass $(n=685)$.

18. Lytle LA, Murray DM, Laska MN, et al. Examining the longitudinal relationship between change in sleep and obesity risk in adolescents. Health Educ Behav. 2013; 40:362-370. [PubMed: 22984211] A longitudinal study examining the change in sleep and change in BMI and body fat; this study found no association across age $14-18$ years $(n=723)$.

19. Mitchell JA, Rodriguez D, Schmitz KH, Audrain-McGovern J. Sleep duration and adolescent obesity. Pediatrics. 2013; 131:e1428-e1434. [PubMed: 23569090] A longitudinal study examining the change in BMI from 14 to 18 years in relation to sleep duration at age 14 years; stronger sleepBMI associations were seen among heavier adolescents. This study is important as it examined BMI change across multiple timepoints in a large cohort $(n=1390)$.

20. Magee CA, Caputi P, Iverson DC. The longitudinal relationship between sleep duration and body mass index in children: a growth mixture modeling approach. J Dev Behav Pediatr. 2013; 34:165173. [PubMed: 23572167] A longitudinal cohort study examining sleep duration and BMI trajectories from early to middle childhood; short sleep may be particularly important in earlyonset obesity, other factors may contribute to late-onset pattern. This study is important as it examined BMI trajectories across time in a large cohort $(n>1000)$.

21. Patel SR, Hayes AL, Blackwell T, et al. The association between sleep patterns and obesity in older adults. Int J Obes. 2014; 38:1159-1164.

22. Miller AL, Kaciroti N, LeBourgeois MK, et al. Sleep timing moderates the concurrent sleep duration-body mass index association in low-income preschool- age children. Acad Pediatr. 2014; 14:207-213. [PubMed: 24602585] Sleep timing magnified the effects of short sleep on BMI zscore in 4-year-old low-income children $(n=273)$; short sleep predicted BMI for children with bedtimes after 9 p.m. and sleep duration was more protective for children with regular weekdayweekend sleep schedules.

23. Scharf RJ, DeBoer MD. Sleep timing and longitudinal weight gain in 4- and 5-year-old children. Pediatr Obesity. 2014 [Epub ahead of print]. In the Early Childhood Longitudinal Birth Cohort Study ( $n=10700$ ), sleep duration at age 4 and 5 years and late bedtime (after 9 p.m.) at 5 years predicted increase in BMI $z$-score and higher odds of obesity.

24. Golley R, Maher C, Matricciani L, Olds T. Sleep duration or bedtime? Exploring the association between sleep timing behaviour, diet and BMI in children and adolescents. Int J Obes. 2013:546551. In a large cohort study of Australian children ( $n=22009-16$ year olds), late-bedtime and late 
rise time pattern was associated with higher BMI $z$-score, higher energy dense dietary intake, independent of sleep duration.

25. Jarrin D, McGrath J, Drake C. Beyond sleep duration: distinct sleep dimensions are associated with obesity in children and adolescents. Int J Obes. 2013; 37:552-558. In two hundred and forty 12year-old children, sleep timing patterns (late bedtime and weekend oversleeping) attenuated the association of sleep duration and adiposity indicators.

26. Adamo KB, Wilson S, Belanger K, Chaput J-P. Later bedtime is associated with greater daily energy intake and screen time in obese adolescents independent of sleep duration. J Sleep Disord Ther. 2013; 2:126-130. Among obese 13 year olds $(n=26)$, a late-sleeping pattern was associated with more calories consumed and more screen time, independent of sleep duration, physical activity, or sedentary behavior.

27. Spruyt K, Molfese DL, Gozal D. Sleep duration, sleep regularity, body weight, and metabolic homeostasis in school-aged children. Pediatrics. 2011; 127:e345-e352. [PubMed: 21262888]

28. Olds TS, Maher CA, Matricciani L. Sleep duration or bedtime? Exploring the relationship between sleep habits and weight status and activity patterns. Sleep. 2011; 34:1299-1307. [PubMed: 21966061]

29. Chung K-F, Kan KK-K, Yeung W-F. Sleep duration, sleep-wake schedule regularity, and body weight in Hong Kong Chinese adolescents. Biol Rhythm Res. 2012; 44:169-179.

30. Kjeldsen J, Hjorth MF, Andersen R, et al. Short sleep duration and large variability in sleep duration are independently associated with dietary risk factors for obesity in Danish school children. Int J Obes. 2014; 38:32-39. Short sleep duration and night-to-night variability was associated with increased energy intake, particularly sugar-sweetened beverages in 676 Danish children (8-11 year olds).

31. Cairns A, Harsh J. Changes in sleep duration, timing, and quality as children transition to kindergarten. Behav Sleep Med. 2013; 12:507-516. [PubMed: 24364713]

32. Roenneberg T, Allebrandt KV, Merrow M, Vetter C. Social jetlag and obesity. Curr Biol. 2012; 22:939-943. [PubMed: 22578422]

33. Baron KG, Reid KJ. Circadian misalignment and health. Int Rev Psychiatry. 2014; 26:139-154. [PubMed: 24892891] This study reviews the circadian misalignment in relation to sleep, eating patterns, and weight gain, methods of assessment, and implications for physical and mental health and disease.

34. Arora T, Taheri S. Associations between late chronotype, body mass index and dietary behaviors in young adolescents. Int J Obes. 2014 [Epub ahead of print]. Among five hundred and eleven 1113 year olds in the UK, evening chronotype and objective-assessed sleep duration were independently associated with higher BMI $z$-score. Chronotype was also associated with lesshealthy dietary intake.

35. Lucassen EA, Zhao X, Rother KI, et al. Evening chronotype is associated with changes in eating behavior, more sleep apnea, and increased stress hormones in short sleeping obese individuals. PLoS One. 2013; 8:6. Among obese adults $(n=119)$, evening chronotype was associated with $\mathrm{BMI}$ increase, eating later in the day, greater cardiovascular risk.

36. Randler C, Haun J, Schaal S. Assessing the influence of sleep-wake variables on body mass index (BMI) in adolescents. Eur J Psychol. 2013; 9:339-347. A self-reported evening chronotype, but not sleep duration, was associated with higher BMI in 13 year olds $(n=913)$.

37. Fleig D, Randler C. Association between chronotype and diet in adolescents based on food logs. Eat Behav. 2009; 10:115-118. [PubMed: 19447353]

38. Kanerva N, Kronholm E, Partonen T, et al. Tendency toward eveningness is associated with unhealthy dietary habits. Chronobiol Int. 2012; 29:920-927. [PubMed: 22823875]

39. Culnan E, Kloss JD, Grandner M. A prospective study of weight gain associated with chronotype among college freshmen. Chronobiol Int. 2013; 30:682-690. [PubMed: 23688114] Among 137 college freshmen, self-reported evening chronotype predicted greater weight gain over 8 weeks.

40. Simpkin CT, Jenni OG, Carskadon MA, et al. Chronotype is associated with the timing of the circadian clock and sleep in toddlers. J Sleep Res. 2014; 23:397-405. [PubMed: 24628737] Among toddler-age children, parent-reported chronotype was associated with dim-light melantonin 
onset, a biological indicator of diurnal preference, suggesting this construct is possible to measure with validity in young children.

41. Taheri S, Lin L, Austin D, et al. Short sleep duration is associated with reduced leptin, elevated ghrelin, and increased body mass index. PLoS Med. 2004; 1:210-217.

42. Boeke CE, Storfer-Isser A, Redline S, Taveras EM. Childhood sleep duration and quality in relation to leptin concentration in two cohort studies. Sleep. 2013; 37:613-620. [PubMed: 24587585] Chronically short sleep was studied in two large cohorts of children $(n=665$ from age 3 to 7 years and $n=502$ from age 16 to 19 years); associations were found between short sleep and lower leptin levels, with some sex differences.

43. Hart CN, Carskadon MA, Considine RV, et al. Changes in children's sleep duration on food intake, weight, and leptin. Pediatrics. 2013; 132:1-8. [PubMed: 23796740] Sleep duration was experimentally manipulated in a small study of 8-11-year-old children $(n=37)$. When sleep was increased, compared with decreased, children consumed fewer calories and had lower leptin levels, weight also decreased.

44. Gonnissen HK, Hulshof T, Westerterp-Plantenga MS. Chronobiology, endocrinology, and energyand food-reward homeostasis. Obes Rev. 2013; 14:405-416. [PubMed: 23387351] A review study on associations among circadian misalignment, energy balance, sleep, meal patterns, and body weight.

45. Garaulet M, Gomez-Abellan P. Timing of food intake and obesity: a novel association. Physiol Behav. 2014; 134:44-50. [PubMed: 24467926] This study reviews the animal and human literature on the timing of caloric intake in relation to adipose tissue regulation and weight loss.

46. St-Onge MP. The role of sleep duration in the regulation of energy balance: effects on energy intakes and expenditure. J Clin Sleep Med. 2013; 9:73-80. [PubMed: 23319909] A review discussing the effects of sleep restriction on energy intake and hormonal changes; this study highlights the methodological discrepancies across the studies that may be responsible for inconsistent findings and calls for tightly controlled studies to assess the behavioral and biological mechanisms.

47. Chaput JP, St-Onge MP. Increased food intake by insufficient sleep in humans: are we jumping the gun on the hormonal explanation? Front Endocrinol. 2014; 5:116. An opinion piece stating the need to consider behavioral pathways, particularly eating behavior, as well as hormonal changes that may explain the association of sleep restriction and increased food intake.

48. Markwald RR, Melanson EL, Smith MR, et al. Impact of insufficient sleep on total daily energy expenditure, food intake, and weight gain. Proc Natl Acad Sci USA. 2013; 110:5695-5700. [PubMed: 23479616] In a small $(n=16)$ experimental sleep restriction study of adults, insufficient sleep resulted in weight gain, primarily because of increased energy intake in the evening.

49. Spaeth AM, Dinges DF, Goel N. Effects of experimental sleep restriction on weight gain, caloric intake, and meal timing in healthy adults. Sleep. 2013; 36:981-990. [PubMed: 23814334] In an experimental study of 225 adults, sleep restriction caused weight gain; greater caloric consumption occurred during evening hours.

50. Nedeltcheva A, Kilkus J, Imperial J, et al. Sleep curtailment is accompanied by increased intake of calories from snacks. Am J Clin Nutr. 2009; 89:126-133. [PubMed: 19056602]

51. Fisher A, McDonald L, van Jaarsveld CH, et al. Sleep and energy intake in early childhood. Int J Obes. 2014; 38:926-929. In the Gemini longitudinal birth-cohort study ( $n=1303)$, parent-reported short sleep at 16 months was related to higher energy intake in infants by 21 months of age (weight status was not related). This is one of the few studies to assess associations this early in the lifespan.

52. Bel S, Michels N, De Vriendt T, et al. Association between self-reported sleep duration and dietary quality in European adolescents. Br J Nutr. 2013; 110:949-959. [PubMed: 23506795] The Healthy Lifestyle in Europe by Nutrition in Adolescence (HELENA) study $(n=1522)$ found crosssectional associations between self-reported short sleep and less-healthy diet.

53. Beebe DW, Simon S, Summer S, et al. Dietary intake following experimentally restricted sleep in adolescents. Sleep. 2013; 36:827-834. [PubMed: 23729925] In this well controlled experimental study $(n=41)$ of sleep restriction using actigraphy to measure sleep and 24-h dietary recalls to assess food intake; 14-16 year olds consumed more sweets in the sleep-restriction condition. 
54. Allison K, Goel N, Ahima R. Delayed timing of eating: impact on weight and metabolism. Curr Obes Rep. 2014; 3:91-100. A review study on late timing of eating as a result of sleep restriction in animal and human literature, and as a mechanism for intervention to enhance weight loss.

55. Garaulet M, Gomez-Abellan P, Alburquerque-Bejar JJ, et al. Timing of food intake predicts weight loss effectiveness. Int J Obes. 2013; 37:604-611. In 420 adult women, greater weight loss was seen among individuals who ate earlier in the day, independent of total caloric intake. In addition, late eaters reported evening chronotype, and CLOCK genotype was related to meal timing.

56. Wang JB, Patterson RE, Ang A, et al. Timing of energy intake during the day is associated with the risk of obesity in adults. J Hum Nutr Diet. 2014; 2:255-262. [PubMed: 23808897]

57. Larson N, Story M. A review of snacking patterns among children and adolescents: what are the implications of snacking for weight status? Child Obes. 2013; 9:104-115. [PubMed: 23470091] Reviews changes in snacking patterns from 2000-2011. Although energy-dense, nutrient-poor snacks are available to most youth, clear associations between snacking and weight status have not been identified. There is a need to better define snacking behavior and the context in which it occurs in order to understand the role of snacking in obesity risk.

58. Eng S, Wagstaff DA, Kranz S. Eating late in the evening is associated with childhood obesity in some age groups but not in all children: the relationship between time of consumption and body weight status in U.S. children. Int J Behav Nutr Phys Act. 2009; 6:1479-5868.

59. Spaeth AM, Dinges DF, Goel N. Sex and race differences in caloric intake during sleep restriction in healthy adults. Am J Clin Nutr. 2014; 100:559-566. [PubMed: 24965304] Adults $(n=44)$ increased their consumption of energy-dense foods when sleep restricted. Male participants gained more weight in the sleep loss condition than did female participants.

60. Baron KG, Reid KJ, Horn LV, Zee PC. Contribution of evening macronutrient intake to total caloric intake and body mass index. Appetite. 2013; 60:246-251. [PubMed: 23036285] In a small study using actigraphy to measure sleep and food logs to measure intake $(n=52)$, late sleepers were found to consume more calories in the evening hours, and higher total calories consumed was associated with BMI above and beyond sleep duration.

61. Braet C, Van Strien T. Assessment of emotional, externally induced and restrained eating behaviour in nine to twelve-year-old obese and nonobese children. Behav Res Ther. 1997; 35:863873. [PubMed: 9299807]

62. Van Jaarsveld CH, Boniface D, Llewellyn CH, Wardle J. Appetite and growth: a longitudinal sibling analysis. JAMA Pediatr. 2014; 168:345-350. [PubMed: 24535222] In the Gemini longitudinal birth-cohort study of 800 twin pairs, parent-reported infant appetite during the first 3 months of life predicted growth patterns at 6 and 15 months. Infants reported as highly foodresponsive and low in satiety responsiveness gained weight more rapidly than their appetitediscordant twin. One of the few studies to examine the associations early in the lifespan.

63. Burt J, Dube L, Thibault L,Gruber R. Sleep and eating in childhood: a potential behavioral mechanism underlying the relationship between poor sleep and obesity. Sleep Med. 2014; 15:7175. [PubMed: 24239496] Shorter sleep duration assessed using actigraphy was related to more obesogenic eating behaviors in a sample of normal-weight children aged 5-12 years $(n=56)$.

64. Dweck JS, Jenkins SM, Nolan LJ. The role of emotional eating and stress in the influence of short sleep on food consumption. Appetite. 2014; 72:106-113. [PubMed: 24148250] 


\section{KEY POINTS}

- Short sleep duration during early childhood is associated with concurrent and later obesity, but little is known about sleep duration-obesity associations in children under the age of 3 years, and associations in adolescents are inconsistent.

- Beyond sleep duration, sleep timing and chronotype may confer unique risk for obesity. Links between sleep timing, chronotype, and obesity risk in children and adolescents are understudied.

- Eating behavior - specifically the timing of eating, nature of dietary intake, and presence of obesogenic eating behavior styles - is a primary behavioral pathway that may confer obesity risk and may be influenced by sleep pattern (duration, timing, and chronotype).

- Longitudinal, experimental, and mechanistic work focused on young children and adolescents is needed to identify the early emergence and trajectories of sleep pattern-obesity risk associations in order to design developmentally appropriate obesity prevention strategies. 